\section{Induction et propagation de la pathologie par la protéine tau chez un modèle murin de la maladie d'Alzheimer}

Florence Clavaguera, Michel Goedert, Markus Tolnay

\section{Pathologie par la protéine tau et dépôts amyloïdes}

Dans le système nerveux central, la protéine tau - constituée de 6 isoformes contrôle la polymérisation des microtubules, composants majeurs des axones impliqués dans le flux axonal et la neurotransmission. Dans de nombreuses maladies neurodégénératives du groupe des tauopathies (dont la maladie d'Alzheimer), la protéine tau est hyperphosphorylée et s'agrège sous forme de filaments intracellulaires [1]. Dans la maladie d'Alzheimer, la tauopathie débute dans les neurones du cortex temporomédian comprenant l'hippocampe, puis s'étend de proche en proche à l'ensemble du cortex cérébral selon un schéma spatiotemporel stéréotypé dont on a montré qu'il corrélait avec le déclin cognitif des sujets affectés [2]. Dans la maladie d'Alzheimer, des dépôts du peptide bêta-amyloïde $(A \beta)$ se forment dans l'espace extracellulaire et s'ajoutent à la pathologie par la protéine tau qui, elle, survient dans les neurones. De nombreux travaux scientifiques ont porté sur la pathologie amyloïde essentiellement à la suite de découvertes de mutations génétiques aboutissant à l'agrégation d'A $\beta$ responsable de cas familiaux précoces de la maladie d'Alzheimer. Plusieurs équipes ont démontré que l'injection d'A $\beta$ dans des cerveaux de souris favorisait le développement de la pathologie de la protéine tau, confirmant ainsi l'hypothèse d'une «cascade amyloïde » $[3,4]$. Toutefois, dans de nombreuses tauopathies, la pathologie par la protéine tau se développe en l'absence totale de dépôts amyloïdes. À la fin des années 1990, la découverte de mutations dans le gène codant pour tau dans des cas familiaux de démence frontotemporale avec parkinsonisme a révélé qu'un dysfonctionnement de tau pouvait être à l'origine d'une dégénérescence neurofibrillaire et d'un déclin cognitif [5-7]. Cette découverte a conduit à la génération de souris transgéniques dont le cerveau présente des lésions intracellulaires constituées de protéine tau humaine [8]. Ainsi, les souris transgéniques P301S (Figure 1), qui expriment la protéine tau humaine mutée (isoforme comprenant 4 domaines répétés mais aucun insert amino-terminal ; 4RON) développent de nombreuses lésions neurofibrillaires constituées de protéine tau hyperphosphorylée (stade tangle $\left.{ }^{1}\right)$ [9]. En revanche, les souris ALZ17 (Figure 1) qui expriment l'isoforme la plus longue de la protéine tau humaine non mutée (isoforme comprenant 4 domaines répétés et 2 inserts aminoterminaux; 4R2N), ne présentent pas d'agrégats de tau et restent à un stade pretangle où tau est hyperphosphorylée mais n'est pas agrégée [10]. Nous avons tiré parti de tels modèles animaux pour étudier les mécanismes sous-jacents à l'induction et à la propagation de la pathologie neurofibrillaire.

\section{La tauopathie fibrillaire peut} se transmettre par voie intracérébrale Pour tester l'hypothèse d'une éventuelle transmission d'une tauopathie

${ }^{1} \varepsilon$ n anglais to tangle signifie enchevêtrer et tangle un imbroglio.
F. Clavaguera, M. Tolnay :

Institute of Pathology,

Department of Neuropathology,

Schönbeinstrasse 40, CH-4031 Basel, Suisse.

F.Clavaguera@unibas.ch

M. Goedert : MRC Laboratory

of Molecular Biology, Hills Road,

Cambridge CB2 OQH, Royaume-Uni.

fibrillaire d'un animal à un autre, nous avons injecté des extraits cérébraux de souris P301S dans le cortex et l'hippocampe de souris ALZ17 âgées de 3 mois (Figure 2A). L'injection d'extraits cérébraux de souris P301S par voie intracérébrale à des souris ALZ17 a provoqué la formation de lésions neurofibrillaires constituées de protéine tau hyperphosphorylée. En effet, 6, 12 et 15 mois après l'injection d'extraits P301S, le marquage argentique Gallyas-Braak de prélèvements cérébraux révélait des lésions fibrillaires et des filaments de tau visibles en immunomicroscopie électronique. En revanche, les animaux non injectés et ceux qui avaient été injectés avec des extraits cérébraux provenant de souris sauvages ou avec des extraits déplétés de protéine tau par immunodéplétion n’ont développé aucune lésion neurofibrillaire. Les structures positives après marquage argentique et reconnues par les anticorps spécifiques de la phosphorylation de tau se sont développées à l'intérieur des cellules cérébrales des souris ALZ17. Ainsi, des lésions morphologiquement identiques à celles des tauopathies humaines se sont développées dans les neurones sous forme de tangles (dans les corps cellulaires neuronaux) et de filaments du neuropile ${ }^{2}$ (dans les prolongements neuronaux), ainsi que dans les oligodendrocytes (corps bobinés) (Figure 2B, C). En microscopie électronique et en immunohistochimie, les filaments étaient décorés par

\footnotetext{
${ }^{2}$ Ou fibres tortueuses.
} 


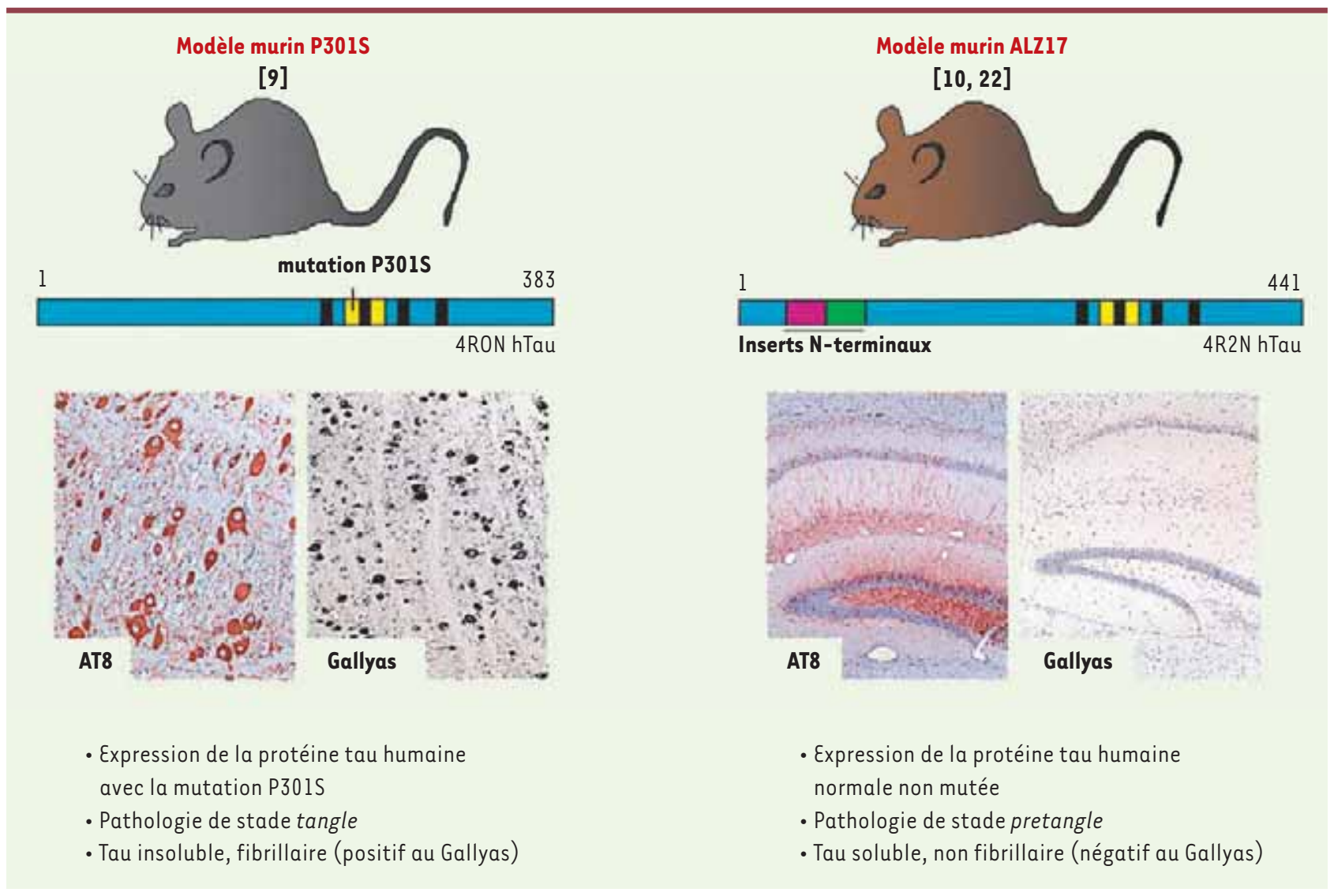

Figure 1. La pathologie de tau chez les souris P301S et ALZ17. Le modèle P301S (à gauche) exprime l'isoforme de la protéine tau humaine qui possède 4 domaines répétés mais pas d'insert amino-terminal. Ce modèle présente une tauopathie sévère au niveau du tronc cérébral et la moelle épinière. L'anticorps AT8 détecte la protéine tau dans un état hyperphosphorylé. Le marquage argentique Gallyas, spécifique de tau agrégé, met en évidence la présence de nombreux tangles. Les photos représentent une coupe coronale du tronc cérébral. Les souris ALZ17 (à droite) sont génétiquement modifiées pour exprimer l'isoforme la plus longue de la protéine tau humaine normale non mutée. Ces animaux présentent une phosphorylation de la protéine tau (marquage AT8 positif) mais pas d'agrégation comme l'indique l'absence de marquage au Gallyas. Les photos représentent une coupe coronale de l'hippocampe.

des anticorps spécifiques des inserts amino-terminaux de tau, démontrant ainsi qu'ils provenaient de la protéine humaine non mutée exprimée par les ALZ17 et non pas des filaments présents dans le matériel injecté. Les lésions neurofibrillaires ainsi induites chez les souris ALZ17 injectées avec l'extrait cérébral de souris P301S évoluaient avec le temps. En effet, l'analyse quantitative a révélé une recrudescence du nombre de lésions positives après marquage Gallyas-Braak entre 6, 12 et 15 mois après I'injection. Cette augmentation semblait dépendre du lieu d'injection, le cortex développant beaucoup moins de lésions positives que l'hippocampe. Nous avons aussi pu mettre en évidence une propagation des lésions positives après coloration argentique depuis les sites d'injection jusqu'à des structures voisines interconnectées anatomiquement comme la fimbria, le tractus optique ou le thalamus. Pour déterminer quelle espèce de tau pathologique était responsable de cette induction neurofibrillaire, la partie soluble de tau et la partie insoluble (constituée d'agrégats de tau) ont été injectées séparément. La partie insoluble de tau, bien que moins abondante que la soluble (Figure 2a), était responsable de la quasi-totalité des lésions neurofibrillaires des souris
ALZ17 injectées avec l'extrait P301S. Nous avons également injecté l'extrait P301S dans le cerveau des souris non transgéniques. Ces animaux ont développé des lésions neurofibrillaires positives par la méthode Gallyas mais de façon modérée. De plus, le nombre de lésions n'augmentait pas avec le temps et l'on n'observait pas de propagation de ces lésions, au contraire des souris ALZ17. En outre, l'analyse des filaments induits a montré qu'ils étaient composés de protéine tau murine et non pas de l'isoforme humaine présente dans l'extrait injecté. Ces résultats suggèrent que l'expression de l'isoforme humaine et/ou la surexpression de tau chez les 


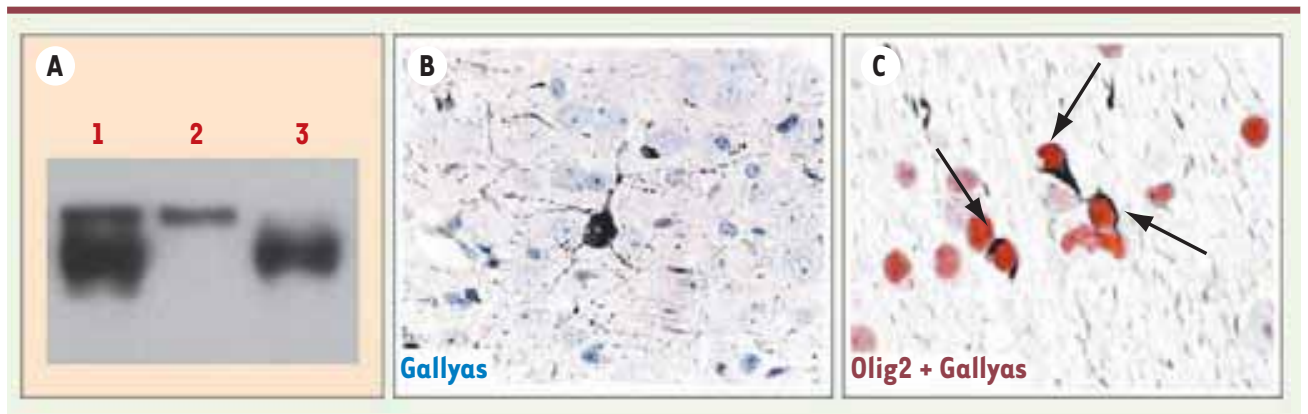

Figure 2. Caractérisation des extraits cérébraux injectés et de la tauopathie fibrillaire induite dans le cerveau des ALZ17. A. Caractérisation par Western-blot (avecl'anticorps HT7 spécifique de la protéine tau humaine) des extraits de souris P301S injectés dans le cortex et I'hippocampe des souris ALZ17. La colonne 1 représente l'extrait total, la colonne 2 la partie insoluble de tau présente dans l'extrait et la colonne 3 la partie soluble de tau de l'extrait P301S. B. Marquage Gallyas détectant la protéine tau sous forme agrégée dans un neurone (ou tangle) de l'hippocampe d'une souris ALZ17 injectée avec l'extrait total de P301S. C. Corps bobinés (flèches) dans la fimbria d'une souris ALZ17 injectée avec de l'extrait P301S. Les oligodendrocytes sont détectés à l'aide de l'anticorps Olig2 (rouge) et le tau fibrillaire par le marquage Gallyas (noir).

souris ALZ17 sont nécessaires pour l'induction de la pathologie neurofibrillaire et sa diffusion en fonction du temps.

\section{En conclusion}

Nos travaux démontrent qu'une transmission expérimentale de la tauopathie neurofibrillaire est possible entre deux lignées de souris transgéniques. Nos résultats indiquent aussi que cette pathologie se propage du site d'injection à d'autres régions cérébrales, ce qui est aussi observé dans la maladie d'Alzheimer. Ce phénomène de conversion de la forme normale de tau au contact de la protéine tau de conformation anormale rappelle le mode de réplication du prion, un agent infectieux composé uniquement de protéine [11]. Un mécanisme similaire a été proposé pour la formation des dépôts du peptide $A \beta$ mais avec cette différence que ce dernier forme des agrégats dans l'espace extracellulaire cérébral et non à l'intérieur des cellules cérébrales [12, 13]. II peut paraître surprenant que l'injection de filaments de tau dans l'espace extracellulaire induise l'agrégation de tau dans les cellules de la souris receveuse. On peut supposer que les agrégats de tau présents dans l'extrait cérébral de souris P30ls sont capables de pénétrer à l'intérieur des neurones et des oligodendrocytes, de les « infecter » et d'y induire la fibrillation de tau endogène. Des études récentes post-mortem de patients atteints de la maladie de Parkinson ayant reçu une greffe de tissu fœtal dopaminergique corroborent nos résultats $[14,15,20]$. Une des observations pathologiques de la maladie de Parkinson consiste en la présence dans les neurones d'inclusions intracytoplasmiques, les corps de Lewy, principalement constitués de la protéine $\alpha$-synucléine agrégée. Le fait que, chez certains patients greffés, des corps de Lewy soient retrouvés à l'intérieur du tissu implanté suggère une propagation de la pathologie de la protéine $\alpha$-synucléine d'un système cellulaire à un autre tout comme dans notre modèle expérimental. Récemment, une étude menée par Desplats et ses collègues met en évidence le passage de la protéine $\alpha$-synucléine d'une cellule à une autre in vitro mais aussi in vivo [16]. À ce jour, plusieurs études in vitro ont montré que des agrégats protéiques ont la propriété de pénétrer dans les cellules et d'y induire, en cascade, l'agrégation de leurs homologues sains. C'est le cas par exemple pour les agrégats de polyglutamines comme ceux qui sont retrouvés dans la maladie de Huntington [17]. Plus proches de notre étude, Frost et ses collègues ont montré que des agrégats extracellulaires de tau pouvaient s'introduire à l'intérieur des cellules et y provoquer la formation de filaments après contact avec tau intracellulaire [18]. Ces observations révèlent des mécanismes moléculaires identiques à ceux des maladies à prions. Cependant, si le mode de réplication de ces protéinopathies paraît similaire, le mode de transmission du prion reste unique et comparable à tout autre agent infectieux. De plus, il est capable d'infecter le système nerveux central d'un individu par voie périphérique alors qu'une étude récente montre que seule l'injection intracérébrale d'extraits $A \beta$ peut transmettre la pathologie. $\varepsilon n$ effet, lorsque ces mêmes extraits sont injectés par voie périphérique (voie orale, intraveineuse, intraoculaire ou intranasale), aucun dépôt de peptide $A \beta$ ne se forme dans le tissu cérébral, la rétine ou le nerf optique [19].

Si toutes les maladies neurodégénératives qui forment des amas protéiques partagent le même mécanisme pathogénique, il est possible d'imaginer un principe thérapeutique commun. Afin de stopper la cascade d'évènements menant à l'agrégation protéique, il semblerait qu'il faille s'attaquer aux agrégats protéiques, les dissoudre, ou les piéger par immunothérapie afin de les empêcher de recruter leurs homologues sains. $\diamond$

Induction and spreading

of tau pathology in a mouse

model of Alzheimer's disease

\section{REMERCIEMENTS}

Nous remercions Monsieur le Professeur Alphonse Probst pour la relecture critique de ce manuscrit. 


\section{CONFLIT D'INTÉRÊTS}

Les auteurs déclarent n'avoir aucun conflit d'intérêts concernant les données publiées dans cet article.

\section{RÉFÉRENCES}

1. Goedert M, Spillantini MG. A century of Alzheimer's disease. Science 2006 ; 314 : 777-81.

2. Braak $H$, Braak $\varepsilon$. Neuropathological stageing of Alzheimer-related changes. Acta Neuropathol 1991 ; 82 : 239-59.

3. Götz J, Chen F, van Dorpe J, Nitsch RM. Formation of neurofibrillary tangles in $\mathrm{P} 301 \mathrm{~L}$ tau transgenic mice induced by Abeta 42 fibrils. Science 2001 ; 293: 1491-5.

4. Bolmont T, Clavaguera F, Meyer-Luehmann M, et al. Induction of tau pathology by intracerebral infusion of amyloid- $\beta$-containing brain extract and by amyloid- $\beta$ deposition in APP $X$ tau transgenic mice. Am J Pathol 2007 ; $171: 2012-20$.

5. Poorkaj P, Bird TD, Wijsman $\varepsilon$, et al. Tau is a candidate gene for chromosome 17 frontotemporal dementia. Ann Neurol $1998 ; 43: 815-25$.

6. Hutton M, Lendon CL, Rizzu P, et al. Association of missense and 5'-splice-site mutations in tau with the inherited dementia FTDP-17. Nature 1998 ; $393: 702-5$.
7. Spillantini MG, Murrell JR, Goedert M, et al. Mutation in the tau gene in familial multiple system tauopathy with presenile dementia. Proc Natl Acad Sci USA 1998 ; 95 : 7737-41.

8. Frank S, Clavaguera F, Tolnay M. Tauopathy models and human neuropathology: similarities and differences. Acta Neuropathol 2008 ; 115 : 39-53.

9. Allen B, Ingram $\varepsilon$, Takao M, et al. Abundant tau filaments and nonapoptotic neurodegeneration in transgenic mice expressing human P301S tau protein. J Neurosci $2002 ; 22: 9340-51$.

10. Probst A, Götz J, Wiederhold KH, et al. Axonopathy and amyotrophy in mice transgenic for human four-repeat tau protein. Acta Neuropathol 2000 ; 99 : 469-81.

11. Prusiner, S.B. Prions. Proc Natl Acad Sci USA 1998 ; $95:$ 13363-83.

12. Kane MD, Lipinski WJ, Callahan MJ, et al. Evidence for seeding of $\beta$-amyloid by intracerebral infusion of Alzheimer brain extracts in $\beta$ amyloid precursor protein-transgenic mice. J Neurosci 2000 ; $15: 3606-11$

13. Meyer-Luehmann M, Coomaraswamy J, Bolmont T, et al. Exogenous induction of cerebral beta-amyloidosis is governed by agent and host. Science 2006 ; $313: 1781-4$

14. Li Jy, Englund $\varepsilon$, Holton JL, et al. Lewy bodies in grafted neurons in subjects with Parkinson's disease suggest host-to-graft disease propagation. Nat Med $2008 ; 14: 501-3$
15. Kordower JH, Chu Y, Hauser RA, Freeman TB, Olanow CW. Lewy body-like pathology in long-term embryonic nigral transplants in Parkinson's disease. Nat Med 2008; 14 : 504-6.

16. Desplats $P$, Lee $H J$, Bae $\varepsilon$ J, et al. Inclusion formation and neuronal cell death through neuron-to-neuron transmission of $\alpha$-synuclein. Proc Natl Acad Sci USA $2009 ; 106: 13010-5$

17. Ren PH, Lauckner JE, Kachirskaia I, et al. Cytoplasmic penetration and persistent infection of mammalian cells by polyglutamine aggregates. Nat Cell Biol 2009 $11: 219-25$.

18. Frost B, Jacks RL, Diamond MI. Propagation of tau misfolding from the outside to the inside of a cell. J Biol Chem 2009; 284 : 12845-52.

19. Eisele YS, Bolmont T, Heikenwalder M, et al. Induction of cerebral beta-amyloidosis: intracerebral versus systemic Abeta inoculation. Proc Natl Acad Sci USA 2009 ; 106 : 12926-31.

20. Langui D, Lachapelle F, Duyckaerts C. Animal models of neurodegenerative diseases. Med Sci (Paris) 2007 ; $23: 180-6$.

21. Lelan F, Damier P. Grafted dopaminergic neurons are targeted by the disease process in Parkinson's disease. Med Sci (Paris) $2009 ; 25: 15-6$.

22. Götz J, Probst A, Spillantini MG, et al. Somatodendritic localization and hyperphosphorylation of tau protein in transgenic mice expressing the longest human brain tau isoform. EMBO J $1995 ; 14: 1304-13$.

\section{Cirs

\section{CNRS Formation Entreprises}

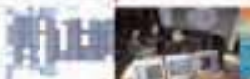

Le 6 mai 2010 Images filtrées en pertes d'énergie d'électrons en microscopie électronique à transmission : de la à PARIS (75) cryomicroscopie à la tomographie électronique en biologie

du 31 mai au 4 juin 2010 Initiation théorique et expérimentale aux techniques de base de la biologie moléculaire à ORSAY (91)

đu 31 mai au 1 juin 2010 Application du dichroïsme circulaire à l'étude des molécules biologiques a GIF SUR YVETTE (91)

du 1 au 3 juin 2010 Ethique, comportement, bien-ètre animal à NOUZILLY (37)

du 2 au 4 juin 2010 Elevage et transgénèse des poissons modèles (poisson-zèbre et médaka) Nouveau à GIF-SUR-YVETTE (91)

du 21 au 25 juin 2010 Initiation théorique et pratique aux techniques de base de la biologie moléculaire

à TOULOUSE (31)

du 21 au 25 fuin 2010 PCR quantitative en temps réel à ORSAY (91)

du 22 au 24 juin 2010 Dépendance aux substances pharmacologiques : Initiation a POITIERS (86) aux techniques d'analyse comportementale chez les petits

Centre de ressources en formation Un problème de formation particulier? Nhésitez pas á nous consulter : - par mail à ressourcesêct.cnrs-git.tr - par téléphone au 01.69.82.44.96 